\title{
Optical properties of tetragonal germanium nanocrystals deposited by the cluster-beam evaporation technique: New light emitting material for future
}

\author{
S NOZAKI*, S SATO, S RATH, H ONO and H MORISAKI \\ Department of Communications and Systems, The University of Electro-Communications, 1-5-1 Chofugaoka, \\ Chofu-shi, Tokyo 182-8585, Japan
}

\begin{abstract}
The germanium (Ge) nanocrystals were deposited on substrates whose temperature was kept at room or liquid nitrogen $\left(\mathrm{LN}_{2}\right)$ temperature by the cluster-beam evaporation technique. The deposited films are found to consist of the tetragonal crystalline structure rather than the diamond structure of bulk Ge. Such a phase-transition has been theoretically predicted for sizes smaller than $4 \mathrm{~nm}$, which agrees with the size measured by the transmission electron microscopy (TEM). The tetragonal Ge is expected to have a direct band gap of $1.47 \mathrm{eV}$. Furthermore, the Ge film deposited at $\mathrm{LN}_{2}$ temperature exhibits unique properties, such as photo-oxidation and blue-light emission. The Ge-nanocrystal films deposited by the cluster-beam evaporation technique are attractive materials for application to light emitting devices in future.
\end{abstract}

Keywords. Germanium; nanocrystal; crystal structure; ST-12; photo-oxidation; blue-light emission.

\section{Introduction}

It is interesting to study the crystalline structure of germanium (Ge) nanocrystals. Saito (1979) reported that the crystalline structures of Ge ultrafine particles, whose diameters were less than $20 \mathrm{~nm}$, were a mixture of the diamond and the tetragonal structures (ST-12). It was theoretically pointed out that nanoparticles with the diamond structure exhibit a phase transition in order to reduce the surface energy when the size becomes smaller than a threshold value. Tomanek and Schluter (1987), for example, predicted a possible phase transition of silicon ( $\mathrm{Si}$ ) nanocrystals from the diamond to body centred cubic (bcc) structure for sizes of $10^{2} \sim 10^{3}$ atoms.

Among the high-pressure forms of $\mathrm{Ge}$, the ST-12 structure is the most attractive for device application. Joannopoulos and Cohen (1973) made a theoretical calculation, using the empirical-pseudopotential method, and predicted that the ST- 12 structure of Ge was a semiconductor with a direct band gap of $1.47 \mathrm{eV}$. The diamond structure of $\mathrm{Ge}$ has been regarded as a less interesting material for device application because of its small and indirect band gap, while the ST-12 structure of $\mathrm{Ge}$ could become an important material for future electronics.

In this study, Ge nanocrystals were deposited by the cluster beam evaporation technique, and their structure and optical properties were studied.

\footnotetext{
*Author for correspondence
}

\section{Experimental}

Figure 1 shows an apparatus of the cluster-beam evaporation technique equipped with a crucible machined from a carbon rod. The cap of the crucible is $1 \mathrm{~mm}$ thick and has a nozzle hole with a diameter of $1 \mathrm{~mm}$. The crucible loaded with small pieces of undoped $\mathrm{Ge}$ $(\sim 40 \Omega \mathrm{cm})$ was heated by electron bombardment. During the deposition, the temperature of the crucible was kept at $2000 \mathrm{~K}$, and the pressure in the chamber was fixed at $5 \times 10^{-6} \mathrm{Torr}$. The substrate temperature was either at room or liquid nitrogen temperature. The typical film thickness was $100 \mathrm{~nm}$ after $60 \mathrm{~min}$ deposition. The classical nucleation theory predicts that the sizes of $\mathrm{Ge}$ nanocrystals formed by the cluster-beam evaporation technique would be much smaller than those by the gas-evaporation technique, which is conventionally used to deposit metal or semiconductor nanocrystals.

$P$-type (100) $\mathrm{Si}$ substrates were used for Raman spectroscopy, electron spectroscopy for chemical analysis (ESCA) and photoluminescence (PL) measurements, while quartz substrates were used for optical absorption measurements. The Raman study was carried out using the $514.5 \mathrm{~nm}$ line of argon (Ar) ion laser with an intensity of $400 \mathrm{~mW}$. The $313 \mathrm{~nm}$ line of a mercury lamp selected with an optical band-pass filter, whose full width at half maximum (FWHM) was $10 \mathrm{~nm}$, was used as an irradiation light and an excitation source for PL measurements. PL and Raman measurements were carried out in the air at room temperature. 


\section{Results and discussion}

Typical Raman spectra of the Ge films deposited at 77 and $300 \mathrm{~K}$ are shown in figure 2, where the Raman spectra of an undoped $\mathrm{Ge}$ wafer $(c-\mathrm{Ge})$ (curve a of figure 2) and a high-pressure form of Ge (ST-12) (curve d of figure 2) are also shown for comparison. The Raman spectra of both Ge films deposited at 77 (curve $c$ of figure 2) and $300 \mathrm{~K}$ (curve $\mathrm{b}$ of figure 2) indicate that their structure is not the ordinary diamond structure but the tetragonal structure, for which Kobliska et al (1972) reported peaks at $273 \pm 3^{-1} \mathrm{~cm}\left(\Gamma_{\varsigma}\right)$ and $246 \pm 3 \mathrm{~cm}^{-1}$ $\left(\Gamma_{3}\right)$. The double peak observed for both Ge films by the cluster-beam evaporation technique resembles those obtained by Kobliska et al (1972). Sato et al (1995)

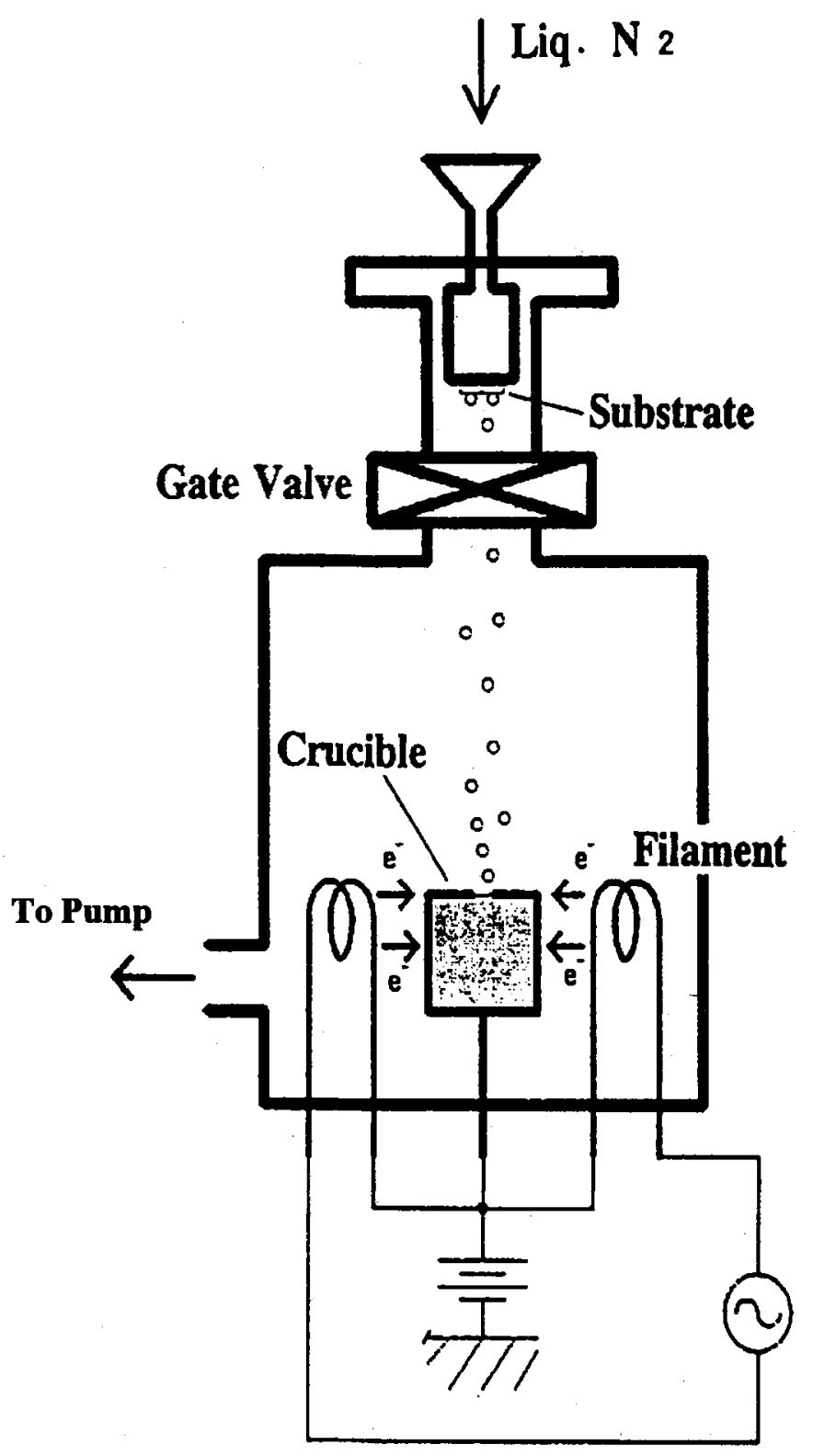

Figure 1. Apparatus of the cluster-beam evaporation technique. reported that in TEM micrographs, the Ge film deposited at $300 \mathrm{~K}$ appeared smooth and uniform, while that deposited at $77 \mathrm{~K}$ was nonuniform and consisted of nanocrystals with diameters ranging from 4 to $40 \mathrm{~nm}$. However, even a larger nanocrystal may be agglomeration of smaller nanocrystals.

Near-infrared to near-ultraviolet optical absorption spectra of the Ge films deposited at 300 and $77 \mathrm{~K}$ are shown in figure 3 (curves $a$ and $b$ ). In the figure, the absorption edge of the film deposited at $300 \mathrm{~K}$ is estimated to be $1.5 \mathrm{eV}$. However, it is difficult to estimate the absorption edge of the film deposited at $77 \mathrm{~K}$ from the spectrum because of no sharp rise in the spectrum. The optical band gap of the Ge film deposited at $300 \mathrm{~K}$ is larger than that of bulk $\mathrm{Ge}(0.66 \mathrm{eV}$, indirect) and close to that of ST-12, predicted by Joannopoulos and Cohen (1973).

All the above results support the presence of the ST-12 structure of $\mathrm{Ge}$ in the $\mathrm{Ge}$ films deposited by the cluster-beam evaporation technique. The detailed study on the crystal structure of the $\mathrm{Ge}$ nanocrystals was reported by Sato et al (1998).

Although the ST-12 structure of Ge is known to be metastable and therefore not stable at high temperature, Nozaki et al (1996) earlier reported the thermally stable ST-12 structure of Ge present in the Ge-nanocrystal films deposited by the cluster-beam evaporation technique. The Raman spectra showed the presence of the tetragonal

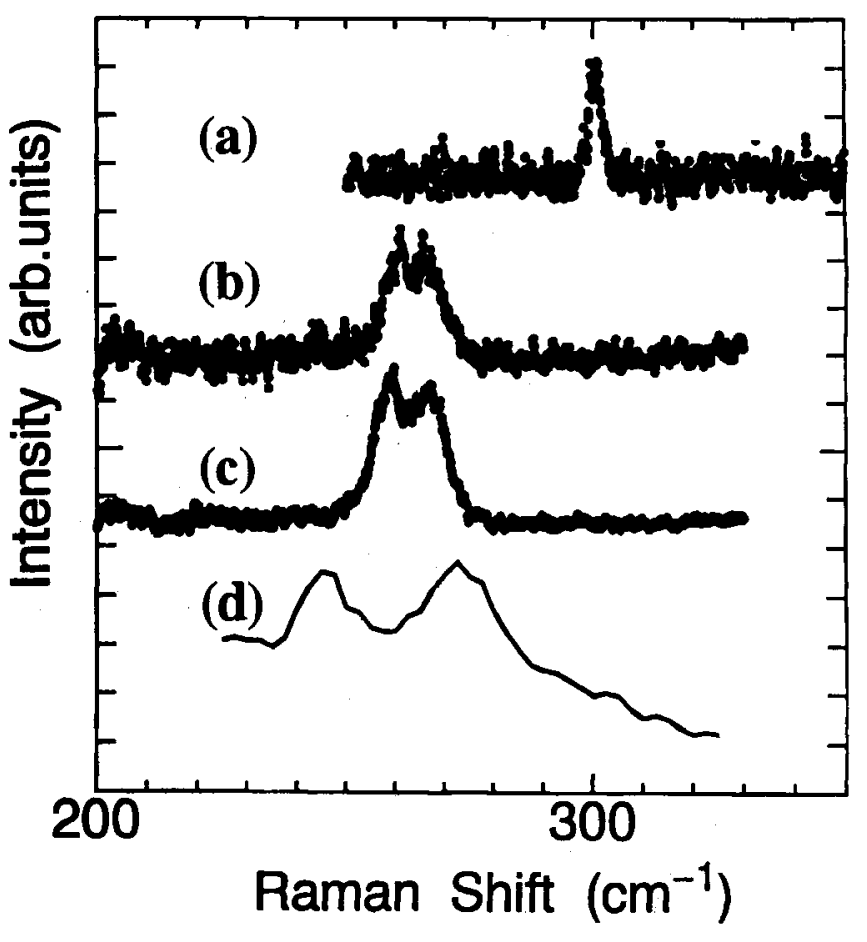

Figure 2. Raman spectra of the Ge films deposited at $300 \mathrm{~K}$ and $77 \mathrm{~K}$ (curves $\mathrm{b}$ and $\mathrm{c}$, respectively). The Raman spectra of bulk Ge and ST-12 structure of Ge are also shown for comparison (curves a and $\mathrm{d}$, respectively). 
structure of $\mathrm{Ge}$ nanocrystals even after annealing at $800^{\circ} \mathrm{C}$.

The ESCA spectra of the surfaces of the Ge films deposited at $77 \mathrm{~K}$ and $300 \mathrm{~K}$ are shown in figure 4 by the curves $a$ and $b$, respectively. The solid and broken curves are the spectra of the as-deposited samples and those of the samples after exposure to $313 \mathrm{~nm}$ line of UV light with a power of $20 \mu \mathrm{W} / \mathrm{mm}^{2}$ for $30 \mathrm{~min}$, respectively. It is observed that the Ge film deposited at $300 \mathrm{~K}$ is hardly oxidized as expected, while the $\mathrm{Ge}$ film deposited at $77 \mathrm{~K}$, which is partially oxidized even before exposure to the UV light, is photo-oxidized rapidly. The photo-oxidation is found to proceed to a deeper level inside the $\mathrm{Ge}$ nanocrystal for a prolonged exposure. With decreasing the size of Ge core, the apparent band gap of a $\mathrm{Ge}$ nanocrystal increases due to the quantum-size effect. An increase in the optical band gap of the $\mathrm{Ge}$ film deposited at $77 \mathrm{~K}$ after photo-oxidation, as shown in figure 3 , curve $c$ evidences the quantum-size effect. The detailed study on photo-oxidation was reported elsewhere by Sato et al (1997).

The observed difference in photo-oxidation of Ge films deposited at 300 and $77 \mathrm{~K}$ cannot be, however, explained merely by a difference in the size. Both sizes are small enough to confine the carriers three-dimensionally. Unlike the film deposited at $77 \mathrm{~K}$, the film deposited at $300 \mathrm{~K}$ is hard to become naturally oxidized and keeps its surface free of oxide, as shown in figure 4 . The film deposited at $77 \mathrm{~K}$ is more subject to natural oxidation perhaps because a larger distance between nanocrystals allows

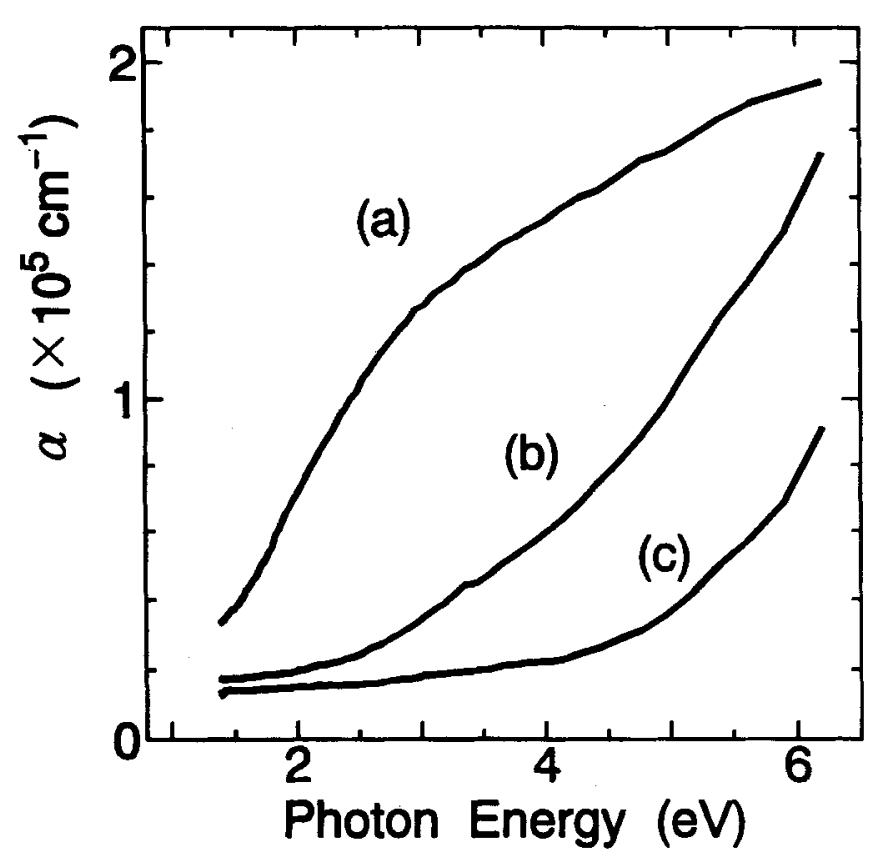

Figure 3. Optical absorption spectra of the Ge films deposited at $300 \mathrm{~K}$ and $77 \mathrm{~K}$ (curves a and $\mathrm{b}$, respectively). The curve c is for the Ge film deposited at $77 \mathrm{~K}$ after exposure to 313 $\mathrm{nm}$ line of UV light with a power of $20 \mu \mathrm{W} / \mathrm{mm}^{2}$ for $30 \mathrm{~min}$. more surface area to be exposed to oxygen. Since the nanocrystals are physically in contact with each other, the carriers in the film deposited at $300 \mathrm{~K}$ cannot be confined in a nanocrystal. A nanocrystal in the Ge-film deposited at $300 \mathrm{~K}$ consisting of several tetragonal-Ge unit cells resembles a grain of a polycrystal. In contrast, a nonocrystal in the Ge film deposited at $77 \mathrm{~K}$ is isolated from the adjacent nanocrystals by the surface oxide barrier. In this case, the carriers are confined threedimensionally, and the quantum-size effect is expected.

According to the model of photonically stimulated oxidation enhancement of Si proposed by Young (1988), the rate-determination step of the oxidation is the dissociation of molecular oxygen into its atoms. In his model, electrons in Si need to be excited to the conduction band of $\mathrm{SiO}_{2}$ in order to dissociate molecular oxygen. Thus, it is quite reasonable to consider that the Ge film deposited at $77 \mathrm{~K}$ shows rapid photo-oxidation but not the film deposited at $300 \mathrm{~K}$, because the conduction band offset at the interface between the $\mathrm{Ge}$ core and the surrounding oxide in the former film is significantly reduced by the three-dimensional carrier confinement. It is also predicted from the photo-oxidation model that the final size of Ge cores is determined by the incident photon energy, and that the size of Ge cores becomes more uniform for prolonged photo-oxidation.

The photoluminescence (PL) spectra of the Ge film deposited at $77 \mathrm{~K}$ after 0,15 , and $30 \mathrm{~min}$ exposures to the UV light are shown in figure 5. The solid curve is a Gaussian curve which is fitted to the experimental

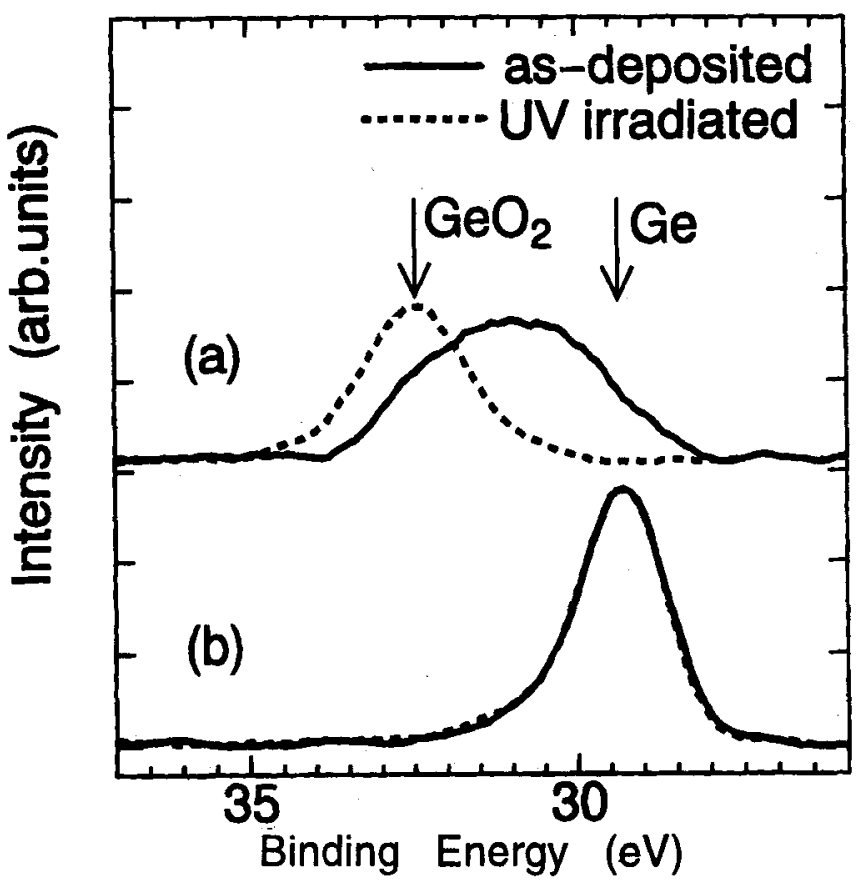

Figure 4. Ge-3d ESCA spectra of the surface of the Ge films deposited at $77 \mathrm{~K}$ and $300 \mathrm{~K}$ (curves a and b, respectively). The solid and broken spectra are for those before and after UV exposure, respectively. 
data. The PL peak energy is $2.8 \mathrm{eV}$, independent of the exposure time. The blue light emission is strong enough to be recognized with the naked eye even under room light. It should be noted that the Ge film deposited at $77 \mathrm{~K}$ shows no detectable luminescence before exposure to the UV light and that the Ge film deposited at $300 \mathrm{~K}$ is not luminescent either before or after exposure to the UV light. Since the PL peak energy is independent of the degree of photo-oxidation, and its intensity correlates with the degree of photo-oxidation, we attribute the blue-light $\mathrm{PL}$ to defects in $\mathrm{GeO}_{x}$.

An effect of anneal on the luminescence characteristic of the $\mathrm{Ge}$ film is more complex. The Ge films deposited at $77 \mathrm{~K}$ were annealed in argon atmosphere at $500^{\circ} \mathrm{C}$ for $60 \mathrm{~min}$. The sequence of photo-oxidation and annealing treatments affects the PL spectrum. A typical PL spectrum of the Ge film after $60 \mathrm{~min}$ UV exposure is shown in figure 6. The PL with a peak energy at $2.8 \mathrm{eV}$ was associated with radiative centres in $\mathrm{GeO}_{x}$, as discussed earlier. The PL spectrum of the Ge film which went through treatments of the $60 \mathrm{~min}$ photo-oxidation and the $60 \mathrm{~min} 500^{\circ} \mathrm{C}$-anneal in that order shows a broader peak with at a PL peak intensity one third of the peak intensity of the photo-oxidized Ge film (curve b of figure 6).

The Ge film which went through the treatments of the photo-oxidation and the anneal in that order shows detectable PL immediately after exposure to the UV light, while the $\mathrm{Ge}$ film without the photo-oxidation treatment before the anneal requires a prolonged exposure to the UV light for detectable PL. The PL spectrum of the latter is not much different from that of the photo-

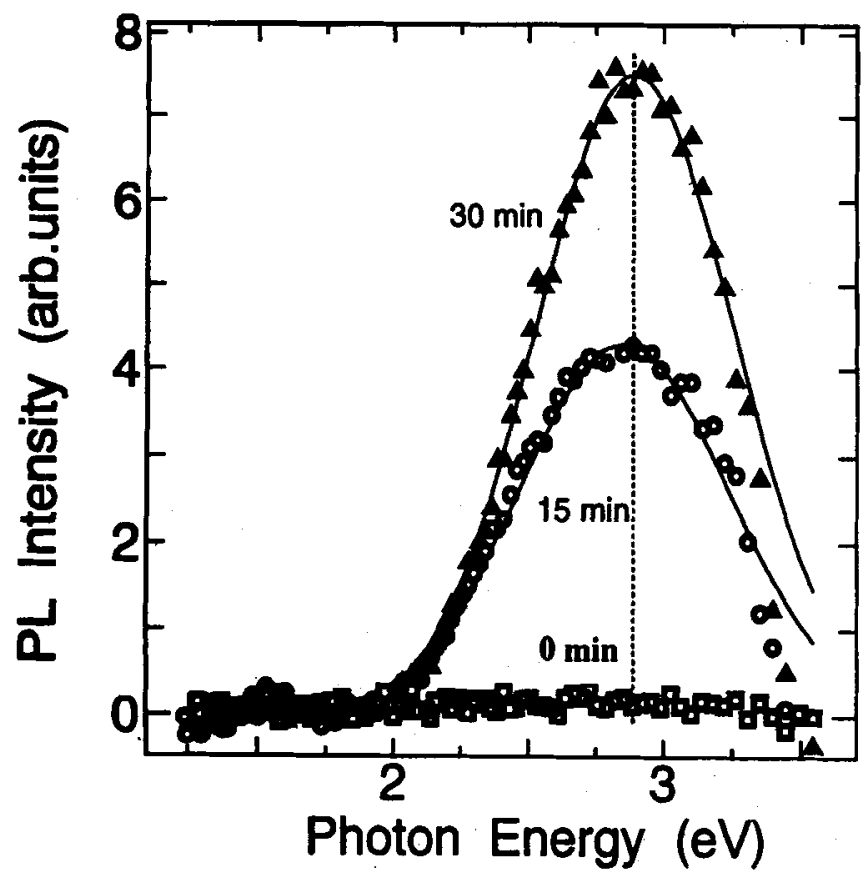

Figure 5. PL spectra of the Ge films deposited at $77 \mathrm{~K}$ after exposure to the UV light for 0,15 and $30 \mathrm{~min}$. oxidized Ge film without the anneal and shows a peak at $2.8 \mathrm{eV}$. Their PL spectra show a constant peak energy and a peak intensity increasing with increasing the UV-exposure time. The effect of the photo-oxidation on the PL spectra overrides that of the anneal.

The above effects of the photo-oxidation and the anneal on the PL are not easily understood. As mentioned earlier, the photo-oxidation of $\mathrm{Ge}$ nanocrystals forms $\mathrm{GeO}_{x}$, where radiative recombination centres are present. The PL with a peak energy at $2.8 \mathrm{eV}$, which contributes to blue-light emission, is attributed to these radiative centres. However, the anneal converts some $\mathrm{GeO}_{x}$ into $\mathrm{GeO}_{2}$ and $\mathrm{Ge}$. This process grows $\mathrm{Ge}$ nanocrystals and decreases the apparent band gap determined by the three-dimensional quantum confinement effect. The PL peak of the Ge film which went through the photooxidation and annealing treatments in that order appears to consist of several peaks including one at $2.8 \mathrm{eV}$. The other peaks may be associated with the $\mathrm{Ge} / \mathrm{GeO}_{2}$ interface or $\mathrm{GeO}_{2}$. The peak at $2.8 \mathrm{eV}$ is attributed to radiative centres in the remaining $\mathrm{GeO}_{x}$.

\section{Conclusions}

Ge films were deposited on substrates whose temperatures were kept at room and liquid nitrogen temperatures by the cluster-beam evaporation technique. The $\mathrm{Ge}$ film deposited at $77 \mathrm{~K}$ is found to consist of nanocrystals

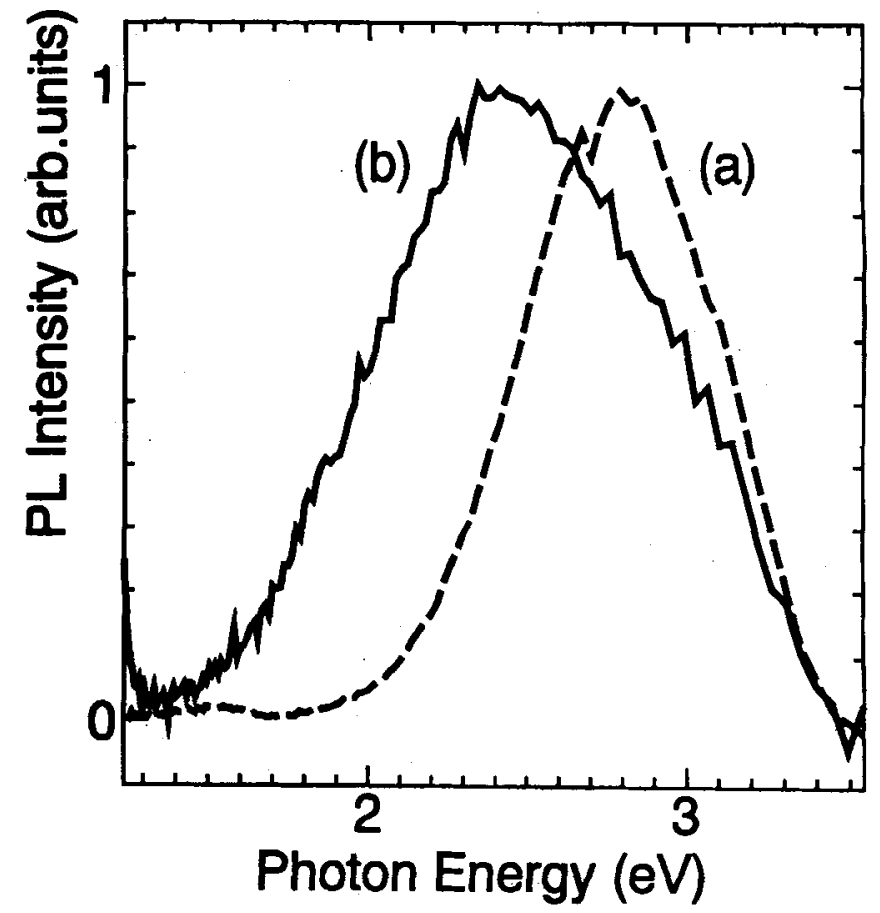

Figure 6. PL spectra of the Ge film deposited at $77 \mathrm{~K}$ after exposure to the UV light for $60 \mathrm{~min}$ (curve a), and the Ge film deposited at $77 \mathrm{~K}$ after exposure to the UV light for 60 $\mathrm{min}$ and subsequent anneal at $500^{\circ} \mathrm{C}$ for $60 \mathrm{~min}$ (curve b). The PL spectrum of the latter was normalized so that the PL peak intensity becomes equal to that of the former. The actual intensity of the latter is one third of that of the former. 
with diameters from 4 to $40 \mathrm{~nm}$, while that deposited at $300 \mathrm{~K}$ appears to consist of much smaller nanocrystals. Even a larger-size nanocrystal in the Ge film deposited at $77 \mathrm{~K}$ may be an agglomeration of smaller-size nanocrystals. Both $\mathrm{Ge}$ films are found to consist of the tetragonal crystalline structure, not the ordinary diamond one. Such a phase transition to a close-packed structure was theoretically expected in order to reduce the surface energy when the size becomes smaller than a critical size. It is also found that the Ge film deposited at $77 \mathrm{~K}$ is oxidized by the UV exposure and consequently exhibits blue light emission. The blue light emission was attributed to defect centres in Ge oxide formed by photo-oxidation. Although the $\mathrm{Ge}$ film deposited at $300 \mathrm{~K}$ is not luminescent in the visible range, it may emit infrared light efficiently, because the ST-12 structure of Ge, which is present in it, is predicted to have a direct band gap of $1.47 \mathrm{eV}$. Therefore, both Ge-nanocrystal films deposited at 300 and $77 \mathrm{~K}$ by the cluster-beam evaporation technique are new attractive materials for light emitting device application.

\section{Acknowledgements}

This study was partly supported by research grants from the Ogasawara Foundation for the Promotion of Science and Engineering, the Mazda Foundation's Research Grant and the Yazaki Science Promotion Foundation, the Special Coordination Funds for Promoting Science and Technology, and by a Grant-in-Aid for Scientific Research, Ministry of Education, Science and Culture.

\section{References}

Joannopoulous J D and Cohen M L 1973 Phys. Rev. B7 2644 Kobliska R J et al 1972 Phys. Rev. Lett. 29725

Nozaki S 1996 Mater. Res. Symp. Proc. 405223

Saito Y 1979 J. Crystal Growth 4761

Sato S et al 1995 Appl. Phys. Lett. 663176

Sato S et al 1997 J. Appl. Phys. 811518

Sato S et al 1998 Appl. Phys. Lett. 722460

Tomanek D and Schluter M A 1987 Phys. Rev. B36 1208 